\title{
Servicios ecosistémicos en la Laguna de Coyuca, Guerrero, México una perspectiva del turismo
}

\author{
Abigail Rosales-Flores* Elizabeth Olmos-Martínez** \\ Investigadora independiente (México)
}

\begin{abstract}
Resumen: Los servicios ecosistémicos son de gran importancia en los territorios costeros, debido a que forman parte del capital natural que se necesita para el desarrollo de la sociedad humana; y también se utilizan para construir escenarios y convertirlos en productos turísticos. El objetivo de ésta investigación es conocer la relación que existe entre la actividad turística y los servicios ecosistémicos de la Laguna de Coyuca y determinar la disposición a pagar del turista para la conservación del hábitat. Se utilizó una metodología mixta en encuestas dirigidas al turista. Los resultados muestran que conocer el lugar y pasear en lancha son el principal motivo de viaje. El turista reconoce los servicios ecosistémicos culturales de la laguna y está dispuesto a pagar una cantidad adicional en los servicios turísticos para la conservación del hábitat de especies bandera mencionadas. Se propone diversificar las actividades turísticas. Lo anterior sirve para la planeación, el aprovechamiento sustentable y la conservación de los recursos naturales locales.
\end{abstract}

Palabras Clave: Servicios ecosistémicos; Servicio ecositémico cultural; Turismo; Laguna costera.

\section{A tourism perpective of ecosystem services in Coyuca Lagoon, Guerrero, México}

Abstract: Ecosystem services are of great importance in the coastal territories, due to they are part of the natural capital that is needed for the development of human society; ecosystem services are also used to build scenarios and turn them into tourist products. The objective of this research is to know the relationship that exists between the tourist activity and the ecosystem services of the Coyuca Lagoon and to determine the willingness to pay of the tourist for the conservation of the habitat. A mixed methodology was used in surveys applied to tourists. The results show that knowing the place and taking a boat ride are the main reasons for traveling to the lagoon. The tourists recognize the cultural ecosystem service of the lagoon and are willing to pay an additional amount for the habitat conservation of the mentioned flag species. The information is usefull for planning sustainable practices, diversifiyng tourism activities and investing adicional resources in the conservation of local natural resources.

Keywords: Ecosystem services; Cultural ecosystem service; Tourism; Coastal lagoon.

\section{Introducción}

El desarrollo y subsistencia de todas las sociedades del planeta, está en función de la relación que el hombre establece con la naturaleza y del grado en el que modifica su entorno natural para obtener bienes y servicios de los cuales se beneficia directa o indirectamente, por lo tanto, los servicios que proporcionan los ecosistemas, hacen posible todas las formas de vida en el planeta (Daily y Ellison, 2002; Gómez-Baggethun y Groot, 2007; Montes y Sala, 2007; Costanza et al., 2011).

No obstante su importancia, los servicios que proporcionan los ecosistemas, no son considerados en el sistema económico actual, pues se identifican como recursos comunes de uso público, como sucede con los paisajes o los valores culturales, que proveen de experiencias intangibles que carecen de un precio y un mercado de consumo específicos (Tallis et al., 2012; Lanza et al., 2013).

\footnotetext{
* Universidad Autónoma de Occidente (México); E-mail: abee.muluc@gmail.com

** Universidad Autónoma de Occidente (México); E-mail: eolmosm.udo@gmail.com; http://orcid.org/0000-0002-7408-0811
} 
Tampoco se integran en la cuentas nacionales la pérdida de biodiversidad, la fragmentación del hábitat, la contaminación u otros efectos negativos que causan diversos problemas ambientales (Balvanera y Cotler, 2007; Camacho-Valdez y Ruiz-Luna, 2012).

En éste contexto, el concepto de servicios ecosistémicos puede vincular la ecología y la economía al reconocer que la conservación de capital natural es fundamental para el desarrollo y bienestar humano (Gómez-Baggethun y Groot, 2007; Labandería et al., 2007), tal como el Gobierno de México establece en del Plan Nacional de Desarrollo 2013-2018: "La conservación del capital natural y sus bienes y servicios ambientales, son un elemento clave para el desarrollo del país y para el bienestar de la población" (PND 2013-2018) (GRM, 2013:77). Al incorporar los elementos del ecosistema a las políticas de desarrollo económico, también se pretende disminuir el deterioro que sufren los ecosistemas (Daily y Ellison, 2002; deGroot et al., 2002; Costanza, et al., 2011).

Los servicios ecosistémicos se definen como los beneficios que las sociedades humanas obtienen de la biodiversidad y de los ecosistemas y se clasifican en cuatro grupos: a) servicios de provisión, b) de regulación, c) culturales y d) de soporte (MA, 2005).

Los servicios de provisión son los productos que se extraen de forma directa, como el alimento y la materia prima (Montes y Sala, 2007; Costanza, et al., 2011); los servicios de regulación se obtienen directamente de los ecosistemas, sin pasar por procesos de transformación, como es el caso del aire limpio, la protección de las costas del impacto de huracanes y tsunamis o la regulación del clima (Corredor et al., 2012; Walters et al., 2008; Sanjurjo et al., 2005); los servicios culturales, forman parte del patrimonio cultural inmaterial y son los beneficios que obtienen las personas a través de experiencias vividas en el ecosistema, como la reflexión, la recreación o el gozo estético (Olivera, 2011; Hernández-Trejo et al., 2012; Angarita-Báez, 2016) y los servicios de soporte, que son los procesos ecológicos que mantienen y hacen posible el resto de los servicios ecosistémicos (Farley y Brown, 2007; Balvanera et al., 2016).

Uno de los elementos que ejerce más influencia medioambiental a la hora de configurar una sociedad y determinar su desarrollo, es la proximidad al mar (Fernández-Armesto, 2002), como sucede en el caso de México, que cuenta con más de $11000 \mathrm{~km}$ de costas y un mar territorial estimado en $231813 \mathrm{~km}^{2}$ (Sarukhán et al., 2009). El ecosistema costero se considera uno de los pilares del desarrollo nacional, ya que soporta actividades económicas como la pesca, la acuicultura, la extracción de hidrocarburos y minerales, el transporte marítimo y el turismo (Contreras y Castañeda, 2004; Morales y Pérez, 2006; Sarukhán, et al., 2009).

Dada la importancia que tienen, los servicios de los ecosistemas costeros son los que han sido más estudiados, en particular los correspondientes a estuarios, lagunas costeras y humedales, debido a que son fundamentales para el mantenimiento de las poblaciones de peces de interés comercial (Sanjurjo et al., 2005; Lara-Lara et al., 2008; Verona-Ezcurra y Rodríguez-Castillo, 2013; Balvanera, et al., 2016), como el atún (Thunnus thynnus, Linnaeus 1758), el camarón (Palaemon serratus, Pennant 1777), la langosta (Panulirus regius, Brito Capello 1864), el pulpo (Octopus vulgaris, Cuvier 1797) y la sardina (Sardina pilchardus, Walbaum 1792), que colocan a México como el productor mundial número 17 en materia pesquera y acuícola, según cifras del Atlas Agroalimentario 2018 (SIAP, 2018).

Además de ser soporte para la actividad pesquera, el territorio costero mexicano destaca porque se ha utilizado para construir escenarios y convertirlos en productos turísticos (Olmos-Martínez et al., 2015; Ortega-Cabrera et al., 2017), como sucedió en Acapulco, Guerrero, el puerto de altura más cercano a la Ciudad de México. Durante mas de 80 años (1932-2012), Acapulco fue planificado para ser el primer destino turístico de sol y playa en México, para logarlo, se aplicaron políticas públicas que excluyeron la participación de la comunidad local y favorecieron a la industria inmobiliaria, que ocupó los espacios con mayor valor paisajístico (Ruz Vargas, 2014).

La industria del turismo se convirtió en la principal fuente de empleo de la entidad y para satisfacer las demandas de un mercado turístico en crecimiento, se cambió el uso de suelo, se deforestaron zonas de manglar y se obstruyeron cauces pluviales, sin considerar los costos socio-ambientales que surgirían en el mediano y largo plazo. Tras su modernización, hoy el puerto de Acapulco vive un declive como destino turístico para viajeros locales e internacionales, debido a la constante urbanización que degrada el ambiente y a la marcada desigualdad que existe en el tejido social, favorecida por el abuso del poder y la dominación de los grupos políticos y económicos dominantes en el país (Ruz Vargas, 2014), además, se ha identificado como un espacio de corrupción e impunidad (Bencomo, 2016). Según señala la Evaluación de Ecosistemas del Milenio (MA, 2005), la degradación de los servicios de los ecosistemas contribuye al aumento de las desigualdades entre los grupos de personas, lo que, en ocasiones, es el principal factor causante de la pobreza y del conflicto social. 
Como la degradación ambiental vulnera la capacidad de generar riqueza en la sociedad (Boege, 2008), es indispensable impulsar proyectos alternativos al turismo de masas que contribuyan a mejorar la calidad y la imagen de los destinos, contrarios al turismo de masas (Huete y Mantecón, 2017), en este contexto, es importante destacar el valor que se le da a un paisaje natural que tiene poco impacto antrópico.

Una estrategia que puede fomentar la conservación del capital natural dentro de la industria turística, es la utilización de especies bandera, término que se utiliza como estrategia de mercado al referirse a especies que son atractivas por su simpatía y captan el interés del público (Isasi Catalá, 2011; Verissimo et al., 2011), éstas especies pueden ser elegidas como símbolo para la protección del ecosistema que representan y a su vez, sirven para recaudar fondos y poder financiar programas y proyectos de conservación (Bowen-Jones y Entwistle, 2002; Ducarme et al., 2013). Aunque el concepto de especie bandera, es parte de la biología de la conservación, considera variables socioeconómicas al vincularse con la mercadotecnia, que es la base para desarrollar una estrategia publicitaria y dirigir las inversiones a proyectos turísticos cuyo objetivo sea salvaguardar el entorno natural de dichas especies (Drumm y Moore, 2002; Verissimo et al., 2011).

Por lo anterior el objetivo de esta investigación es conocer la relación que existe entre la actividad turística y los servicios ecosistémicos de la Laguna de Coyuca, Guerrero y determinar la disposición a pagar del turista por la conservación del hábitat.

\section{Descripción de los Municipios de Acapulco de Juárez y Coyuca de Benitez, estado de Guerrero en el Pacífico Mexicano.}

El estado de Guerrero, es considerado el cuarto con mayor biodiversidad de México (detrás de Chiapas, Oaxaca y Veracruz), se ubica en la Costa del Pacifico y una de sus principales actividades económicas es el turismo de sol y playa (Botelloa et al., 2015). El desarrollo intensivo de ésta actividad se inició en la década de 1930, cuando Acapulco fue conocido como el Paraíso de América y se convirtió en el primer puerto turístico internacional y destino de playa del país; durante la década de 1940, artistas de Hollywood convirtieron esta bahía mexicana en uno de sus principales lugares de descanso y en una pieza central en la nueva definición sobre el turismo en México (Velázquez, 2013). Acapulco fue estratégicamente planeado como destino vacacional para captar inversiones y aprovechar la belleza de un paraíso "salvaje" todavía por civilizar, se desarrolló a partir de la construcción de hoteles y casas "modernas"; las inversiones federales en infraestructura urbana crecieron, pero se concentraron en la zona costera, convirtiendo al puerto en una fachada turística que no consideró los costos socio-ambientales que generaría en el mediano y largo plazo (Velázquez, 2013; Ruz-Vargas, 2014).

El gobierno del municipio de Acapulco de Juárez divide geográficamente la actividad turística en cuatro zonas: Tradicional, Dorada, Diamante y Pie de la Cuesta (Centro de Documentos Turísticos (2011), éste último se ubica diez kilómetros al noroeste de Acapulco y se distingue por el fuerte oleaje del Océano Pacífico, la puesta del sol, los paseos a caballo en la playa y los recorridos en lancha por la Laguna de Coyuca, durante los cuales los guías relatan historias y anécdotas del lugar (Anzaldúa-Soulé, 2013). Los recursos naturales que integran el paisaje de Pie de la Cuesta, ofrecen la oportunidad de generar un nuevo aprovechamiento turístico en Acapulco, desarrollando actividades que transmitan las características estéticas, geográficas, históricas y culturales del paisaje, desde una perspectiva sustentable (Castillo-Elias, 2010; Anzaldúa-Soulé, 2013) ya que el paisaje natural del lugar, contrasta con la infraestructura hotelera de las otras tres zonas turísticas.

Conviene destacar que el paisaje de la Laguna de Coyuca, se convierte en un bien de consumo para el turismo que visita la zona y por lo tanto, se crea una dependencia económica del patrimonio natural (Aguilar Aguilar et al., 2015). Dado lo anterior, el objetivo de esta investigación es conocer el perfil del turista que visita la Laguna de Coyuca, sus patrones de consumo, los motivos que lo llevan a visitar el lugar, sus gustos y preferencias por otras actividades recreativas, y la relación que tienen con los servicios ecosistémicos que presta la laguna. Lo anterior es una herramienta para planear el desarrollo turístico de tal manera que los residentes locales tengan mayor participación en los beneficios económicos que genere la actividad turística y se sientan motivados a conservar los atributos ambientales del lugar. 


\section{La Laguna de Coyuca}

La Laguna de Coyuca es una de las siete lagunas costeras con bocas efímeras que desembocan en la vertiente del Océano Pacífico (Piedragil y Mejía, 2001), se ubica entre los Municipios de Acapulco de Juárez y Coyuca de Benítez (Fig. 1), tiene una superficie aproximada de $34 \mathrm{~km}^{2}$, con longitud de $10.6 \mathrm{~km}$, ancho promedio de $2.8 \mathrm{~km}$ y una línea de costa de $26.7 \mathrm{~km}$ (José Luis Rosas-Acevedo, 2014). Se considera un sitio prioritario para la conservación de la biodiversidad del país y está dentro de la lista de humedales de importancia a nivel nacional (CONABIO, 2009). La Comisión Nacional para el Conocimiento y Uso de la Biodiversidad (CONABIO), reporta la existencia de 144 especies de vertebrados e invertebrados que viven en el sistema lagunar Coyuca-Mitla (Flores-Verdugo F., 2007) . Como recurso forestal, los manglares de la laguna (Rhizophora mangle, Laguncularia racemosa y Conocarpus erectus), aportan beneficios sociales, económicos y culturales a doce comunidades localizadas a menos de 100mts, que subsisten con los productos que obtiene de la pesca, la extracción de madera y las actividades turísticas que se desarrollan en el lugar (Sanjurjo y Welsh, 2005; Flores-Verdugo F., 2007; Calderón et al., 2009; Castillo-Elias, 2010).

Figura 1: Localización de la Laguna de Coyuca, Guerrero

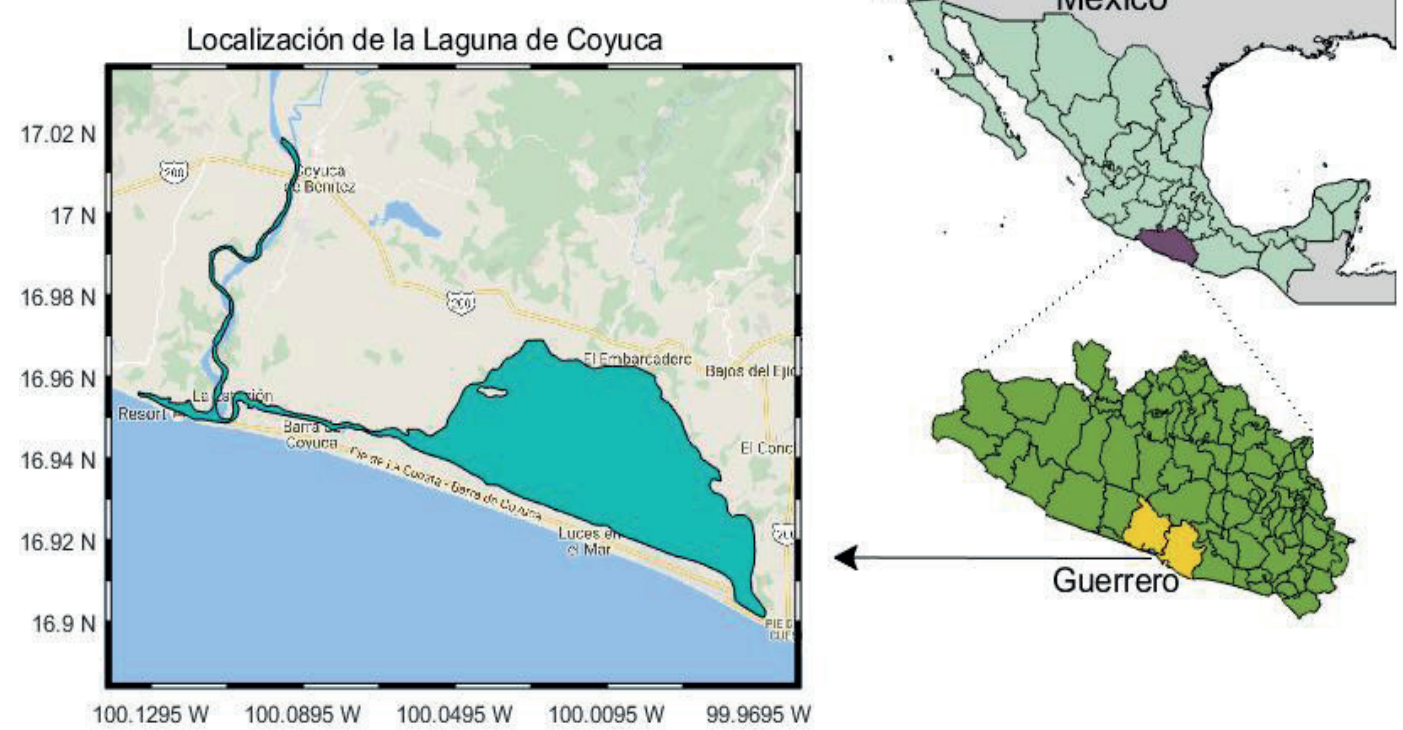

Fuente: elaboración propia

\section{Materiales y métodos}

De acuerdo con los antecedentes expuestos, la presente investigación es descriptiva, debido a que se observan los fenómenos tal y como ocurren sin intervenir en su desarrollo y describe los datos y las características de los sujetos de estudio a partir de las costumbres, procesos, actitudes y situaciones predominantes mediante la utilización de datos cualitativos y cuantitativos (Hernández Sampieri et al., 2014).

Los datos cualitativos describen la realidad tal como es observada por los actores, en este caso, se busca tener una aproximación de la relación que existe entre los turistas y los servicios ecosistémicos que brinda la Laguna de Coyuca, y hacer una descripción de los motivos y preferencias que tienen por ciertas actividades recreativas en un contexto de turismo de naturaleza. 
Los datos cuantitativos permiten analizar la disposición a pagar que tienen los turistas con el fin de destinar los recursos a realizar acciones de conservación de la laguna y, con los datos que proporcionen, describir el perfil socioeconómico de los visitantes.

El instrumento de recolección de datos es una encuesta que consta de 24 preguntas, divididas en cuatro secciones: 1) datos generales, 2) información sobre el viaje, 3) experiencia en la laguna y 4) percepción y conocimiento de los servicios ecosistémicos.

Se incluyeron los cuatro servicios ecositémicos: el servicio de regulación de perturbaciones (protección que ofrecen los manglares contra tormenta y huracanes) y el servicio de provisión (peces y camarones como alimento), se abordaron desde la educación ambiental, con una pregunta cerrada que afirma o niega las aseveraciones con respecto a los bienes y servicios que proporcionan los manglares. El servicio de soporte se consideró en una pregunta dicotómica para conocer la disposición que tiene el turista de pagar una cuota destinada a conservar el hábitat de las especies bandera que se mencionaron: el cocodrilo (Crocodylus acutus, Cuvier 1807), la tortuga endémica pecho quebrado (Kinosternon integrum, Le Conte 1854) y la guacamaya verde (Ara militaris, Linnaeus 1766), sujetas a protección especial según la NOM -059-SEMARNAT-2010 (DOF, 2010).

El servicio ecosistémico cultural se abordó desde la perspectiva de las actividades recreativas que son posibles desarrollar en la laguna, y que, de desarrollarse, ofrecerían una alternativa de turismo de naturaleza y de bajo impacto, se solicitó que las actividades fueran ordenadas jerárquicamente para identificar las preferencias que tienen los turistas y se ofreció un rango de precios para conocer el monto que están dispuestos a pagar por recibir el servicio.

Para determinar el tamaño de la muestra, se tomó el número total de turistas que llegaron a Acapulco en 2014, debido a que no hay un conteo específico para el área de Pie de la Cuesta, los datos se tomaron del Anuario Estadístico y Geográfico de Guerrero 2015, que reportó la llegada de 4,590,910 personas. De acuerdo con las técnicas de muestreo para estudios sociales (Calero, 1978), se consideró un nivel de confianza del $95 \%$ y un error de estimación del 5\% obteniendo como resultado una muestra de 384.

En enero de 2017, el diseño de la encuesta fue evaluado con una prueba piloto aplicada a 70 personas y durante febrero y agosto de 2017 se aplicaron las 384 encuestas en el embarcadero de Pie de la Cuesta y en la Isla Montosa.

El testimonio y las observaciones adicionales que hicieron los turistas fueron registrados en un diario de campo y se creó un expediente fotográfico.

\section{Resultados perfil del turista}

El perfil del turista que visita la Laguna de Coyuca, tiene una proporción de mujeres de 53\% ( $\mathrm{n}=384$ ), mayor que la de los hombres (47\%). El promedio de edad de las mujeres que visitaron la Laguna de Coyuca, fue de 39 años y el de los hombres de 36 años. La edad mínima de los encuestados fue 18 y la máxima de 78 años. El rango de edad más frecuente fue entre 18 y 45 años, en el que se ubicó el 71\% de los turistas.

En cuanto a sus características socioeconómicas: el ingreso mínimo mensual reportado fue de $\$ 1,000$ y el máximo de $\$ 40,000$ pesos, y un promedio de $\$ 9,073$ pesos mensuales; el ingreso que mayor número de veces se repitió fue $\$ 10,000$ pesos. El 31\% $(n=384)$ de la muestra reportó tener un rango de ingresos entre $\$ 1,000$ y $\$ 5,000$ pesos y el $30 \%$ se ubicó en el rango de $\$ 5,001$ y $\$ 10,000$ pesos, esto indica que el $61 \%$ de los encuestados se encuentra en un rango de ingresos mensuales entre $\$ 1,000$ y $\$ 10,000$. Cabe señalar que, el 18\% de los encuestados no contestó la pregunta, a pesar de que se informó sobre el anonimato y confidencialidad de los datos.

La mayoría de los turistas, viajó desde la Ciudad de México (42\%) y el Estado de México (28\%) (Fig.2), dos grandes zonas metropolitanas que se encuentran a no mas de $400 \mathrm{~km}$ de distancia de Acapulco; además, se encuestó a una persona extranjera que llegó a la Isla Montosa, desde Canadá. 
Figura 2: Lugar de procedencia de los turistas que llegan a la Laguna de Coyuca

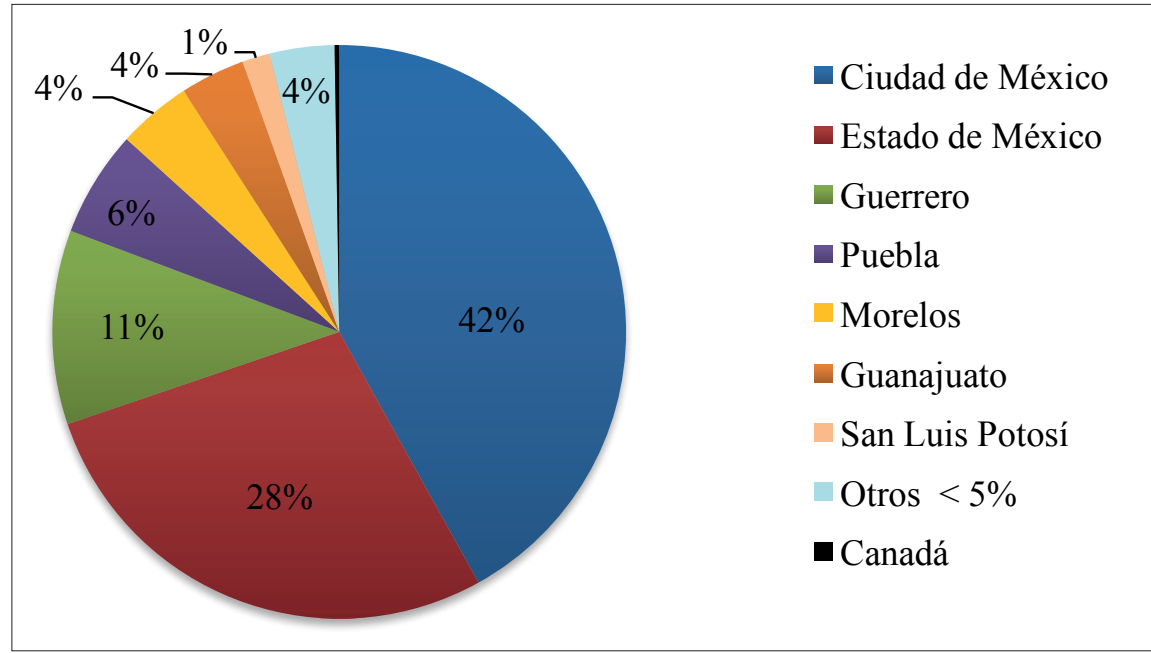

Acapulco es la playa más cercana a la zona metropolitana que emite el mayor número de turistas, la principal via de acceso para el $97 \%(n=384)$ de las personas que llegaron a la Laguna de Coyuca, fue la terrestre; el 59\% utilizó el automóvil particular para su traslado y el 38\% viajó en autobús, sólo el 2\% que utilizó taxi o motocicleta, se desplazó desde algún lugar del municipio de Acapulco o de Coyuca de Benítez. El 1\% de la población que utilizó avión, viajó desde Coahuila, Mazatlán y Canadá.

El 51\% (n=384) de los turistas encuestados, viajó en grupo de dos a cinco personas y el $25 \%$ lo hizo en grupo de hasta diez personas, quienes viajaron en grupos mayores a 16 personas, fueron aquellos que visitaron la laguna como parte del itinerario de una excursión a Acapulco y representaron el 10\% de la muestra. Cabe destacar que el $72 \%$ de las personas que visitaron la Laguna de Coyuca, lo hizo por primera vez.

\section{Resultados sobre experiencia y preferencias}

Para conocer la satisfacción de los usuarios en el viaje a la Isla Montosa, se hizo una pregunta cerrada con tres opciones de respuesta, el 83\% (n=384\%) respondió que la experiencia fue buena, el 15\% regular y el $2 \%$ mala. Las nueve personas que consideraron tener una experiencia mala, dijeron que no volverían a visitar la Laguna de Coyuca, y entre las razones que mencionaron estuvieron: la mala atención y desorganización por parte de los prestadores de servicios, porque se sintieron engañados con la información que recibieron previo al viaje en lancha o porque consideraron que el lugar no tiene la infraestructura básica en servicios sanitarios y de atención médica.

Para saber qué tanto se inclinan los turistas por elegir las lagunas costeras como un sitio recreativo que favorece el contacto con la naturaleza, se hizo una pregunta que considera la Laguna de Tres Palos como opción de visita, además de la Isla de la Roqueta, las playas de Acapulco o alguna alternativa sugerida por la persona encuestada; los resultados muestran que el 68\% (n=384) de los turistas prefirió la playa, reafirmando que Acapulco se identifica como un destino de sol y playa, mientras que la Laguna de Tres Palos fue elegida por el 5\% de la muestra. Tanto la Laguna de Coyuca como la de Tres Palos, son promocionadas en la Guía Turística 2015-2018 que publica la Secretaría de Turismo del Gobierno Municipal de Acapulco (2015), donde son descritas como lugares donde se puede practicar deportes acuáticos, hacer un recorrido por los manglares y observar aves.

\section{Relación del turista con los servicios ecosistémicos de la Laguna de Coyuca}

En cuanto al conocimiento y percepción del turista sobre el servicio ecosistémico de regulación y protección que proporciona el ecosistema de manglar en la Laguna de Coyuca, el 71\% (n=384) de 
los turistas, respondió que es verdadero que los manglares sirven de protección contra tormentas y huracanes (Tabla 1).

Considerando que la Laguna de Coyuca es también una fuente importante de suministro de alimento para la población y que el pescado y los camarones, son los principales platillos que se ofrecen en los dos restaurantes que dan servicio en la Isla Montosa, se preguntó a los turistas si tenían el conocimiento de que el manglar es el hábitat necesario para que estas especies se desarrollen en las primeras etapas de su vida y poderlas consumir después, destaca que el $81 \%$ de los encuestados sí sabía que se beneficia de éste servicio proporcionado por el ecosistema de la laguna costera (Tabla 1).

Tabla 1: Laguna de Coyuca: Conocimiento de los servicios ecosistémicos, 2017

\begin{tabular}{lcccc}
\hline Servicio ecosistémico & Verdadero & Falso & No sé & Total \% \\
Regulación & 71 & 17 & 12 & 100 \\
Provisión & 80 & 8 & 11 & 100 \\
\hline
\end{tabular}

Fuente: elaboración propia

El servicio ecosistémico cultural más representativo de la Laguna de Coyuca, es el turístico-recreativo, siendo los paseos en lancha a la Isla Montosa, la actividad más difundida y una de las principales fuentes de ingreso para la comunidad. Las actividades que se consideraron en la encuesta como alternativa turísticas de bajo impacto que además pudieran promover el conocimiento y la conservación de la biodiversidad entre los turistas, fueron: 1) los senderos interpretativos con torres para la observación de aves, 2) criaderos de tortugas y/o cocodrilos y 3) un parque temático, 4) paseos nocturnos por la laguna, 5) eventos gastronómicos con comida típica, 6) eventos musicales, 7) práctica de yoga y 8) celebraciones religiosas.

De entre las ocho opciones de actividades recreativas propuestas, los encuestados sólo debían elegir cuatro y ordenarlas jerárquicamente por orden de preferencia. Los resultados indican que los criaderos de tortugas y cocodrilos son los preferidos, siendo elegidos como la primera y segunda opción por el 31\% y $27 \%(n=384)$ respectivamente. El paseo nocturno por la Laguna de Coyuca, fue elegido como la tercer opción por el $24 \%$ y en cuarto lugar seleccionaron el festival de música, representado por el $20 \%$ de la muestra. Cabe señalar que los senderos interpretativos y las torres para observar aves, fue la segunda actividad que los usuarios eligieron como primer opción, siendo representada por el 18\% (Fig.3).

Figura 3: Orden de preferencia de las actividades recreativas propuestas

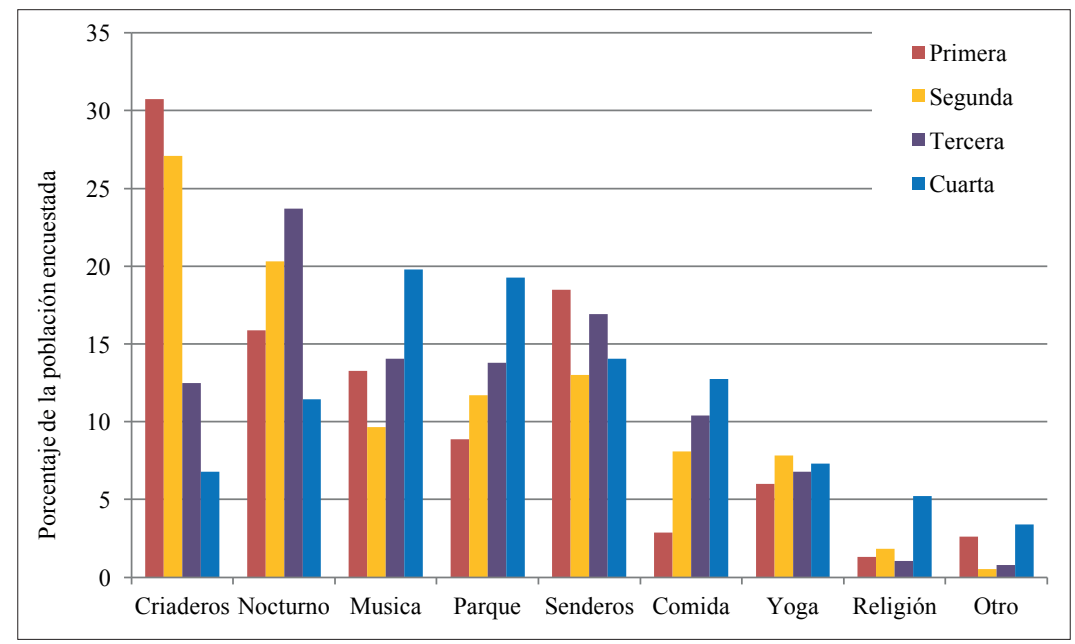

Fuente: elaboración propia 


\section{Presupuesto para la actividad recreativa}

Para conocer cuál es el presupuesto que asignarían los turistas para gastar en alguna de las actividades recreativas elegidas, suponiendo en este caso, cuánto estarían dispuestos a gastar por entrar a un criadero de tortugas y/o cocodrilos; se dieron cinco opciones de respuesta, que van de menos de $\$ 50$ pesos hasta más de $\$ 200$ pesos. El $41 \%$ se ubicó en el rango que va de $\$ 50$ a $\$ 100$ pesos, seguido por el $16 \%$ con un rango que va de $\$ 101$ a $\$ 150$ pesos (Figura 4). El 5\% de los usuarios respondió que el pago dependía del servicio o de algunos factores que consideraron importantes, como el número de personas que viajan juntas, los servicios adicionales ofertados durante la visita, o el presupuesto familiar asignado para el viaje.

Figura 4: Presupuesto asignado para actividad recreativa

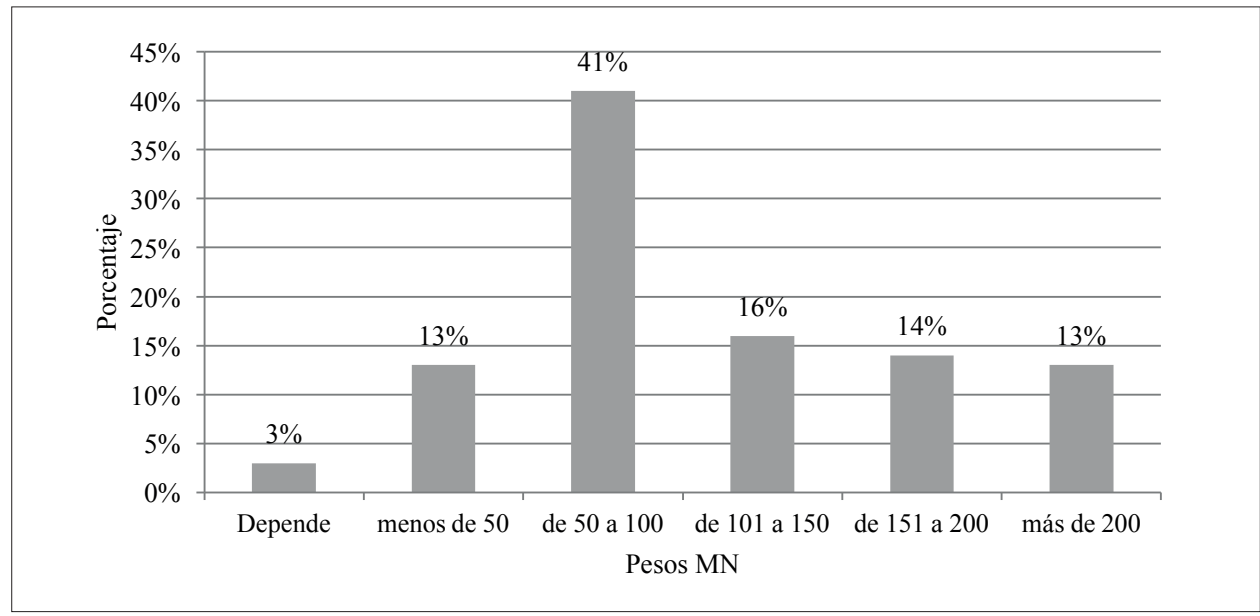

Los resultados muestran que, para la mayoría de los turistas, un precio aceptable por realizar una actividad recreativa diferente en la Laguna de Coyuca, sería de hasta $\$ 100$ pesos, el equivalente al precio que pagan por viajar en lancha a la Isla Montosa.

\section{Disposición a pagar una cuota de conservación}

En cuanto a la disposición a pagar una cuota para que se destine a acciones de conservación del hábitat de las especies bandera, el 91\% (n=384) de las personas encuestadas contestó que si estaban dispuestos a pagar una cantidad adicional a la que pagaron por viajar en lancha a la Isla Montosa para tal fin. La cantidad adicional fue definida por los usuarios, la mínima que establecieron, fue de $\$ 10$ pesos y la máxima de $\$ 500$ pesos, ambas representadas por el 14\% $(n=351)$ de las personas que sí estuvieron de acuerdo con un incremento en el costo de viaje en lancha (Fig.5). El 53\% de los turistas, estuvo dispuesto a aportar hasta $\$ 50$ pesos, seguido por el $26 \%$ dispuesto a pagar hasta $\$ 100$ pesos adicionales a lo que pagaron por el viaje en lancha (Fig.5).

Del total de personas encuestadas, solo $9 \%(n=384)$ no estuvo dispuesta a pagar ninguna cantidad adicional, para la conservación del hábitat. Entre los motivos por los cuales se negaron a realizar dicha contribución, está el que considera que la conservación de los recursos es responsabilidad del Estado $(24 \%, n=33)$, señalando en algunos casos a la Secretaría de Turismo (SECTUR) y a la Secretaría de Medio Ambiente y Recursos Naturales (SEMARNAT) (Tabla 2), como las dependencias gubernamentales que deberían vigilar y encargarse de que la laguna esté en buen estado para que se puedan realizar actividades recreativas. En concordancia con las personas que señalaron al Estado como responsable de la conservación de los recursos naturales, el 6\% $(n=33)$ señaló que la administración pública municipal debe obtener recursos para la conservación, por medio de algún impuesto que sea destinado al cuidado de la naturaleza. Estos resultados indican que para el 30\% de los turistas que no estuvieron dispuestos a pagar, el cuidado, administración y conservación de la Laguna de Coyuca como ecosistema, es responsabilidad del Estado. 
Figura 5: Presupuesto asignado para actividad recreativa

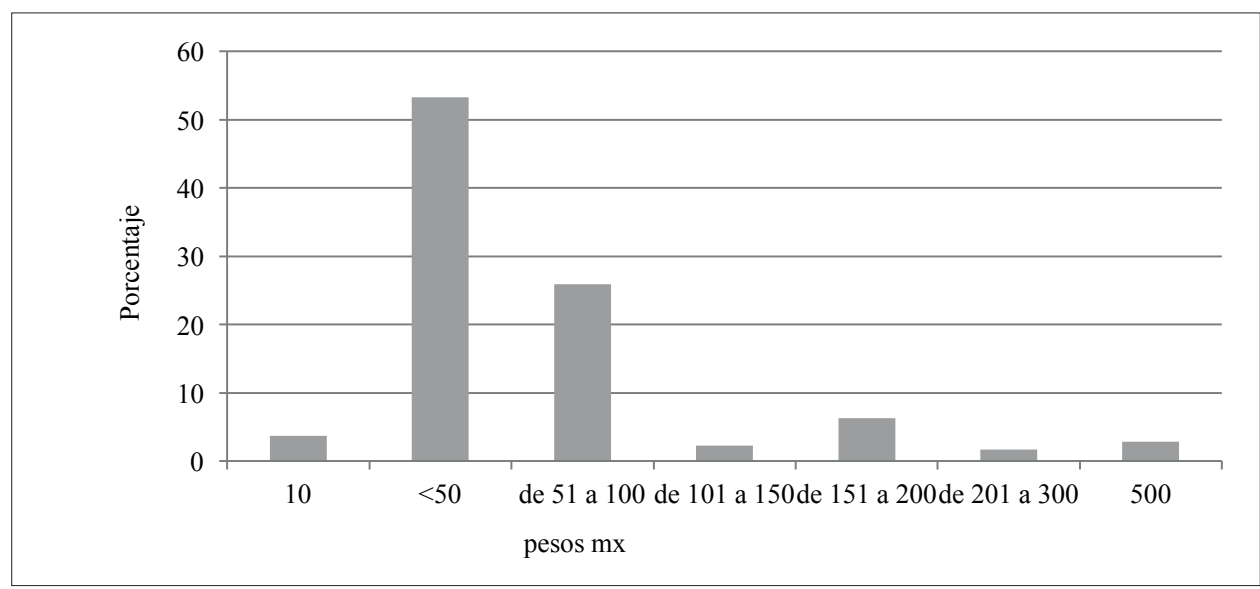

Nueve de las treinta y tres personas $(9 \%, \mathrm{n}=33)$ que no pagarían más, señalaron que los turistas son responsables del deterioro del medio ambiente y por lo tanto, cualquier cuota necesaria para el mantenimiento del hábitat, debe ser incluida en el precio del boleto y el monto recaudado, debe ser administrado por los prestadores de servicios o por la comunidad, pues en su opinión, son quienes se benefician directamente de los servicios ecosistémicos que provee la Laguna de Coyuca.

Un factor importante que restringe la disposición a pagar para la conservación, es la falta de solvencia económica, como lo manifestó el $24 \%$ (n=33) y también es importante señalar que el $36 \%$ de las personas que no estuvieron dispuestas a contribuir económicamente para la conservación del hábitat, mostró indiferencia y se deslindó de cualquier responsabilidad con el medio ambiente.

Tabla 2: Motivos para negar un pago adicional para la conservación

\begin{tabular}{|l|c|c|}
\hline \multicolumn{1}{|c|}{ Motivo } & No. Individuos & Porcentaje \\
\hline Es responsabilidad del Estado & 8 & 24 \\
\hline Que se recaude a través de un impuesto & 2 & 6 \\
\hline Debe incluirse en el precio del boleto & 3 & 9 \\
\hline No tengo solvencia económica & 8 & 24 \\
\hline No es mi responsabilidad & 6 & 18 \\
\hline Sin explicación & 6 & 18 \\
\hline Total & 33 & $100 \%$ \\
\hline
\end{tabular}

\section{Análisis del perfil del turista}

Las variables socieconómicas muestran que los turistas que viajan a la Laguna de Coyuca, son personas adultas con una edad promedio de 35 a 40 años, que tienen un ingreso promedio mensual de $\$ 9,073$ pesos mensuales y la mayoría $(48 \% \mathrm{n}=384)$ son originarios de la Ciudad de México $(\mathrm{CdMx})$ y Estado de México (28\% n=384).

Éstos resultados indican que Acapulco sigue siendo un «destino turístico preferencial» para el turismo nacional, especialmente para la Cdmx y área metroolitana, por su cercanía geográfica y porque tiene oferta para todos los niveles socioeconómicos (Solís-Radilla et al., 2016), no obstante, son las capas sociales con bajos ingresos las que visitaron la Laguna de Coyuca. Para Cerón y Lechuga (2018) el turismo social es una opción de turismo para la población de ingresos modestos, es decir, aquellas familias cuyo ingreso mensual es de entre $\$ 7,122$ y $\$ 11.446$ pesos, que se caracteriza por financiarse con fuentes informales (tandas, empeño, cajas de ahorro, préstamos a la palabra) y programan sus 
viajes a través de empresas unipersonales o pequeñas tanto formales como informales, en las que se les ofrecen paquetes de viaje sencillos. Cabe señalar que el 15.8\% de la población del país cuenta con ese nivel de ingreso (Cerón y Lechuga, 2018), por lo que ésta información es importante para el análisis de las políticas públicas del sector turístico de México.

No obstante los turistas que visitaron la Laguna de Coyuca tienen un ingreso modesto, el $79 \%$ ( $\mathrm{n}=384)$ estuvo dispuesto a pagar hasta $\$ 100$ pesos adicionales para acciones de conservación del ecosistema, esto significa un incremento del $100 \%$ a la tarifa actual que cobran los prestadores de servicios por el paseo en lancha. De generarse los ingresos adicionales, podrían ser destinados a proyectos turísticos de bajo impacto que preserven el capital natural de la Laguna de Coyuca.

\section{Análisis del motivo de la visita}

De todas las decisiones que tiene que tomar el turista potencial, la elección del destino vacacional es la más importante (Pons et al., 2007). Para cualquier individuo, la decisión de viajar se basa en sus necesidades y deseos, no obstante, en el proceso de toma de decisiones, la relación imagen-destino es un factor determinante, ya que, la imagen de un destino turístico es resultado de la percepción y actitud que se tenga del entorno (Heung et al., 2001; Pons, et al., 2007).

La actitud, las experiencias pasadas y las expectativas del lugar son elementos que se consideran al elegir un destino de viaje (Lam y Hsu, 2006). Algunos estudios sobre turismo indican que la diversión, la exploración y la comida son los motivos más comunes al momento de elegir un destino e indican qué tan atractivo puede ser un lugar para satisfacer la expectativa que tiene el turista (Heung et al., 2001).

Elegir un lugar de destino implica un proceso complejo de toma de decisiones, y entender cómo los turistas deciden qué lugar visitar, permite establecer modelos de comportamiento del consumidor y desarrollar estrategias de mercado apropiadas (Heung, et al., 2001; Pons, et al., 2007; Ai-Naa et al., 2016), para lo que es necesario considerar todos los elementos del destino, no sólo la imagen (Murphy et al., 2007).

En el caso de la zona turística de Pie de la Cuesta, donde se ubica la Laguna de Coyuca, existe un estudio realizado en 2013 por Anzaldúa-Soulé, quien reportó que el 39\% (n=385) de los visitantes que llegaron a Pie de la Cuesta, fueron motivados por el paisaje y la naturaleza del lugar, en ese sentido la tabla 3 muestra la comparación del motivo del visitante del presente estudio y del realizado en 2013 , se observa que existe una gran diferencia en los motivos pasados y presentes ya que para 2013, tal como lo menciona la autora "la demanda turística del sitio se inclina por los paisajes y el contacto con la naturaleza, por lo que, sería importante conservar el área y crear conciencia en la población y en los prestadores de servicios para gestionar acciones de conservación" (Anzaldúa-Soulé, 2013: p. 93). No obstante, es posible que el turista de 2017 no considere el paisaje y la naturaleza de la laguna como el principal motivo para visitarla, lo anterior puede beberse a diferentes factores donde el tipo de información social, económica y ambiental influye en las preferencias e intereses, o bien, a que existen diferencias entre quienes llegan a Pie de la Cuesta y quienes visitan específicamente la Laguna de Coyuca.

Tabla 3: Comparativo de los motivos del viaje 2013 - 2017

\begin{tabular}{|l|c|l|c|}
\hline \multicolumn{2}{|c|}{ Pie de la Cuesta, 2013 } & \multicolumn{2}{c|}{ Laguna de Coyuca, 2017 n=384 } \\
\hline \multicolumn{1}{|c|}{ Motivo } & Porcentaje & \multicolumn{1}{c|}{ Motivo } & Porcentaje \\
\hline Ocio, recreación y descanso & 31 & Conocer & 34 \\
\hline Paisaje & 22 & Recomendación & 14 \\
\hline Naturaleza & 17 & Familia y amigos & 8 \\
\hline Salud & 9 & Promotor & 8 \\
\hline Cultura & 7 & Excursión & 7 \\
\hline Amigos y Familia & 5 & Paisaje y laguna & 6 \\
\hline Compras & 4 & Descanso & 6 \\
\hline Estudios & 3 & Vacaciones & 6 \\
\hline Negocios & 2 & Por gusto & 4 \\
\hline & & Diversión & $100 \%$ \\
\hline Total & $100 \%$ & & \\
\hline
\end{tabular}

Fuente: elaboración propia con datos de Anzaldúa-Soulé (2013) y de la encuesta aplicada en la Laguna de Coyuca (2017). 


\section{Servicio ecosistémico cultural y actividad turística de bajo impacto}

Los resultados indican que los criaderos de tortugas y cocodrilos y los senderos interpretativos con torres para observar aves, son las dos actividades que más llamaron la atención de los turistas. Respecto a los criaderos de tortugas, en el periódico oficial del Gobierno del Estado de Guerrero se estableció la necesidad de "salvaguardar, conservar y promover la supervivencia y calidad de vida de las tortugas marinas, dotando de equipamiento a campamentos tortugueros y promoviendo la expansión de proyectos productivos en ese rubro, con el fin de mantener vigente el derecho humano al medio ambiente sano" (EG,(2016, pp. 7-8). Mientras que en Acapulco, por medio de la Guía Turística de Acapulco (2015) se promueve la liberación de tortugas golfinas (Lepidochelys olivácea, Eschscholtz,1829) de octubre a enero y en un campamento tortuguero en Playa Larga.

Cabe destacar que las personas que administran los dos restaurantes en la Isla Montosa de la Laguna de Coyuca, tienen en cautiverio un cocodrilo (Crocodylus acutus) y algunas tortugas endémicas "pecho quebrado" (Kinosternon integrum), especies sujetas a protección especial de acuerdo con la Norma Oficial Mexicana NOM-059-SEMARNAT 2010 (DOF, 2010). Los animales sobreviven dentro de un estanque pequeño, sucio y con poca agua, además de que no hay ningún letrero que de información sobre las especies y su hábitat; en éste sentido, $6 \%(n=384)$ de las personas que viajaron a la Isla Montosa, señalaron el cautiverio como un problema ambiental. Esta situación contradice la acción de fomentar el trato humano a los animales y promover la conservación de la biodiversidad, para proteger el patrimonio natural, señalado como estrategia en el Plan Nacional de Desarrollo 2013-2018 para impulsar un crecimiento verde y generar riqueza.

Sobre los senderos interpretativos, la Norma Mexicana NMX-AA-133-SCFI-2006, publicada en el D.O.F. 14/03/2006, establece que un sendero es un pequeño camino que permite recorrer con facilidad un área determinada y tiene la función de servir de acceso para los visitantes, pero además, de acuerdo con la Secretaría de Turismo, sirven para estimular el interés de los visitantes para conocer la relación que existe entre los seres humanos y el medio ambiente a través de un proceso de reflexión (SECTUR, 2004).

Éstas dos actividades requieren de infraestructura de baja impacto y son compatibles con el entorno natural, misma que se puede aprovechar para combinar la recreación y la educación ambiental, además de generar una derrama económica en la población, tal como lo señala.

\section{Conclusiones}

La riqueza cultural de una sociedad, está estrechamente ligada al conocimiento, uso y aprovechamiento de los ecosistemas en los cuales se desarrolla (Boege, 2008), para éste caso de estudio, se encontró que la mayoría de los turistas reconocen que existe una estrecha relación entre su bienestar y el estado en el que se encuentra el ambiente del destino seleccionado. Tal como lo menciona el concepto de servicios ecosistémicos ya que alude a que el valorar los beneficios que recibe la sociedad por los bienes y servicios que obtiene directa o indirectamente de la naturaleza, ayuda a integrar los elementos de la naturaleza al momento de decidir la planeación de destinos turísticos sustentables con beneficios para las comunidades receptoras.

En el caso de la Laguna de Coyuca, se utilizó el servicio ecosistémicos de soporte que brinda el manglar para que fuese reconocido como hábitat de tres especies sujetas a protección especial y pudiera ser valorado en términos monetarios con la disposición a pagar por parte del turista. Los resultados demostraron que es posible generar recursos financieros para dedicarlos a la conservación, no obstante, es importante llevar a cabo una la gestión inteligente y eficiente de los recursos, esto puede lograrse con una planificación adecuada de las actividades recreativas donde se involucren los actores sociales y gubernamentales en la toma de decisiones, complementado con un centro de información donde se implemente un programa de educación ambiental para que los turistas puedan conocer el entorno y disfrutarlo. De lo contrario sucede lo que Esquivel-Ríos et al. (2014) comentan y está relacionado con que, el diseño de proyectos turísticos destinados a la conservación del ambiente, ha sido cuestionado en diferentes ámbitos, debido a la escasa participación de las comunidades locales, la carencia de información para la toma de decisiones, la ausencia de personal capacitado y en la mayoría de los casos, la falta de recursos para su implementación.

De acuerdo con Strand (2000) los mecanismos basados en el mercado para la gobernanza y gestión de recursos naturales, han modificado su enfoque, orientándose hacia la valoración de los ecosistemas como medida adecuada de conservación para lograr la debida atención, utilizando todos los instrumentos 
necesarios que le permitan a las instituciones gubernamentales abordar los desafíos ambientales de manera práctica y efectiva. En el caso de la laguna de Coyuca es necesario que el Gobierno estatal y municipal implemente estrategias para el turismo responsable donde se valore los servicios ecosistémicos como un elemento intrínseco de la actividad.

Por otro lado, se considera que el identificar a la Laguna de Coyuca con una especie bandera, serviría para desarrollar una estrategia de mercado que capte la atención de los turistas y genere los recursos financieros necesarios para conservar e integrar los servicios ecosistémicos a los planes de desarrollo de lugar. Se propone utilizar una especie bandera, porque además de sus atributos biológicos, son consideradas por los valores culturales y sociales que propician, ya que permite que la comunidad se identifique con el patrimonio natural (Isasi Catalá, 2011; Verissimo et al., 2011; Ducarme, 2013).

Además, la participación de la comunidad con los proyectos turísticos que se desarrollen, en el destino, ayudará aconservar el capital natural de la Laguna de Coyuca, en ese sentido, el paisaje de la laguna es un recurso turístico de gran valor para un sector del mercado que demanda experiencias diferentes a la que caracteriza el turismo tradicional que se ofrece en la Bahía de Acapulco. El paisaje poco urbanizado de la laguna, es apreciado por los turistas que proceden de la Ciudad de México, quienes están acostumbrados a vivir en un paisaje urbano-industrial, un espacio congestionado por el tráfico vehicular y degradado por la contaminación atmosférica y acústica. De acuerdo con Cohen (2005), en éste tipo de experiencia de contraste de paisajes, reside la emoción que motiva al turista a desplazarse y es precisamente la expectativa de emocionarse lo que atrae nuevos perfiles de turistas. Para la Lagua de Coyuca, se espera atraer un segmento turístico que se defina por el deseo de aproximarse a la naturaleza desde el respeto y la voluntad de preservación del ambiente.

El sector turístico es sensible en sus relaciones con el medio ambiente, ya que necesita un entorno natural atractivo para su desarrollo y la percepción ambiental que se tenga de él, condiciona e influye considerablemente en las actitudes y preferencias de los visitantes (Figueiredo et al., 2012; deUña-Álvarez et al., 2017; Huete y Mantecón, 2017). Por ejemplo, en sitios turísticos donde el paisaje natural es uno de los elementos centrales del viaje, como sucede en la Laguna de Coyuca, las actividades recreativas de bajo impacto que utilizan la interpretación del paisaje como herramienta de educación ambiental, son la mejor alternativa para integrar la naturaleza al tiempo de ocio y recreación que destina el turista en el lugar, así lo demuestra éste estudio, al señalar los criaderos de tortugas y/o cocodrilos y los senderos interpretativos con torres para observar aves, como las actividades más atractivas y por las cuales los turistas estuvieron dispuestos a pagar una cantidad adicional para su conservación.

De ésta manera, el turismo puede ayudar a la conservación del ecosistema donde se realiza, puede contribuir al desarrollo económico de las comunidades y conservar los atributos ambientales del lugar, cuando la actividad recreativa, además provee de una experiencia educativa e incorpora el uso racional de los recursos naturales (Hsieh et al., 2004; Clifton y Benson, 2006), ya que la experiencia muestra que las actividades recreativas no planificadas, traen consecuencias no deseadas para los ecosistemas acarreando pérdida de bienes y servicios ambientales indispensables para el desarrollo y el mantenimiento de las sociedades humanas (Viñals-Vlasco et al., 2011).

Finalmente, las limitaciones del presente estudio permiten recomendar a futuros investigadores un acercamiento real con las autoridades, además de generación de talleres para la comunidad, ya que es necesario que, no solo los turistas valoren los beneficios de los servicios ecosistémicos, sino que, la comunidad receptora sea el principal actor local en en generar acciones concretas para su conservación, ya que de ello depende la dinámica de la actividad turística en la región. El alcance de ésta investigación es local y/o municipal, no obstante, sienta un precedente para continuar con estudios que complementen los resultados y que coadyuven conjuntamente para la toma de decisiones para el desarrollo de la actividad turística, sobre todo por la necesidad imperante debido a la cercanía con el puerto turístico de Acapulco ya que son los turistas que llegan a dicho puerto los que visitan la Laguna de Coyuca, su cultura, gastronomía y sus servicios ambientales.

\section{Bibliografia}

Aguilar Aguilar, A., Palafox Muñoz, A., y Anaya Ortiz, J. S. 2015. El turismo y la transformación del paisaje natural. Nóesis. Revista de Ciencias Sociales y Humanidades, 24, 19-29.

Ai-Naa, S., Yuen-Onnb, C., y Ling-Meng, C. 2016. Travel Intentions among Foreign Tourists for Medical Treatment in Malaysia: An Empirical Study. Procedia - Social and Behavioral Sciences 224, 546 - 553 
Angarita-Báez, J. 2016. Servicios ecositémicos culturales del Territorio indígena del Corregimiento La Pedrera, Amazonas-Colombia Maestrá en Desarrollo Sustentable y Gestión Ambiental, Universidad Distrital Francisco Jose de Caldas Bogotá, Colombia.

Anzaldúa-Soulé, K. R. 2013. El aprovechamiento turístico del paisaje desde una visión sustentable: una propuesta para Pie de la Cuesta, municipio de Acapulco de Juárez, Guerrero Maestría en administración e innovación del turismo, Instituto Politécnico Nacional, México, D.F.

Balvanera, P., Armendáriz-Arnez, C., y Tauro, A. 2016. Los servicios ecosistémicos. In P. Balvanera, E. Arias-González, R. Rodríguez-Estrella, L. Almeida-Leñero \& J. Schmitter-Soto (Eds.), Ecosistemas de México: una mirada a su conocimiento (pp. 229-339). Ciudad de México: Universidad Nacional Autónoma de México.

Balvanera, P. y Cotler, H. 2007. Acercamiento al estudio de los servicios ambientales. Gaceta Ecológica, 84-85, 88-15.

Bencomo, A. 2016. Acapulco: del tropicalismo a la distopía urbana. Telar, 17, 25-37.

Boege, E. 2008. El patrimonio biocultural de los pueblos indígenas de México (1 ed.). México, D.F.

Botelloa, F., Sánchez-Corderoa, V., y Ortega-Huerta, M. A. 2015. Disponibilidad de hábitats adecuados para especies de mamíferos a escalas regional (estado de Guerrero) y nacional (México). Revista Mexicana de Biodiversidad 86, 226-237.

Bowen-Jones, E. y Entwistle, A. 2002. Identifying appropriate flagship species: the importance of culture and local contexts. Oryx, 36(2).

Calderón, C., Aburto, O., y Ezcurra, E. 2009. El valor de los manglares. Biodiversitas, 82

Calero, A. 1978. Técnica de muestreo. La Habana: Editorial Pueblo y Educación.

Camacho-Valdez, V. y Ruiz-Luna, A. 2012. Marco conceptual y clasificación de los servicios ecositémicos. Bio Ciencias, 1(4), 3-15.

Castillo-Elias, B. 2010. Diagnóstico ambiental del maglar en la Laguna de Coyuca de Benítez, Guerrero, México. Doctorado en Ciencias Ambientales, Universidad Autónoma de Guerrero, Acapulco, Guerrero.

CentroDocumentosTurísticos. 2011. Plan Sectorial de Desarrollo Turístico de la Zona Metropolitana de Acapulco. In F. N. d. F. a. Turismo (Ed.), (Vol. Disponible en [1CD-ROM]. ). México DF. .

Cerón, H. y Lechuga, M. 2018. Análisis de los hábitos de viaje del turismo social en México, específicamente, del segmento de bajos ingresos. Turismo y Sociedad, XXIV, 101-128.

Clifton, J. y Benson, A. 2006. Planning for sustainable ecotourism: the case for research ecotourism in developing country destinations. Journal of Sustainable Tourism, 14(3), 238-254.

Cohen, E. 2005. Principales tendencias en el turismo contemporáneo. Política y Sociedad, 42(1), 11-24.

CONABIO. 2009. Sitios de manglar con relevancia biológica y con necesidades de rehabilitación ecológica. México DF: CONABIO.

Contreras, F. y Castañeda, O. 2004. La biodiversidad de las lagunas costeras. Ciencias, 76, 46-56.

Costanza, R., Kubiszewski, I., Ervin, D., Bluffstone, R., Boyd, J., Brown, D., . . Yeakley, A. (2011). Valuing ecological systems and services. Biology Reports 2011, 3(14).

Daily, G. y Ellison, K. 2002. The New Economy of Nature. The Quest to Make Conservation Profitable. Washington, DC: Island Press.

deGroot, R., Wilson, M., y M.JBoumans, R. 2002. A typology for the classification, description and valuation of ecosystem functions, goods and services. Ecological Economics, 41(2), 393-408.

deUña-Álvarez, E., Cuquejo-Bello, M. d. C., y Villarino-Pérez, M. 2017. Valoración local del patrimonio natural para el desarrollo turístico en un territrio rural transfronterizo (Sierra del Larouco, Galicia-Norte de Portugal). Papeles de Geografía, 63.

DOF, D. O. d. 1. F. 2010. NORMA Oficial Mexicana NOM-059-SEMARNAT-2010. Protección ambiental-Especies nativas de México de flora y fauna silvestres-Categorías de riesgo y especificaciones para su inclusión, exclusión o cambio-Lista de especies en riesgo. (Jueves 30 de diciembre de 2010).

Drumm, A. y Moore, A. 2002. Desarrollo del Ecoturismo. Un manual para los profesionales de la conservación. The Nature Conservancy, 1(88).

Ducarme, F., Luque, G. M., y Courchamp, F. 2013. What are "charismatic species" for conservation biologists? BioSciences Master Reviews, July.

Esquivel-Ríos, S., Cruz-Jiménez, G., Cadena-Inostroza, C., y Zizumbo-Villarreal, L. 2014. El turismo como instrumento de política ambiental en el Santuario de la Mariposa Monarca El Rosario. Sociedad y Territorio, XIV(4), 141-174.

EstadodeGuerrero(EG). 2016. Periódico Oficial del Gobierno del Estado de Guerrero. Chilpancingo, Gro.: Retrieved from http://periodicooficial.guerrero.gob.mx/wp-content/uploads/2016/03/PERIODICO-18.pdf. 
Farley, J. y Brown, E. 2007. Restoring Natural Capital: An Ecological Economics Assessment. In J. Aronson, S. J. Milton \& J. N. Blignaut (Eds.), Restoring natural capital : science, business, and practice. Washington: Island Press.

Fernández-Armesto, F. 2002. La lista de los elementos que componen una civilización. In S. E. Generales (Ed.), Civilizaciones, la lucha del hombre por controlar la naturaleza (pp. 44-47). España: Taurus.

Figueiredo, S. L., Nóbrega, W., Bahia, M., y Piani, A. 2012. PPlanificación y gestión de las visitas al patrimonio natural y cultural y a los atractivos turísticos. Estudios y Perspectivas en Turismo, 21, 355-371.

Flores-Verdugo F., M.-C. P., Agraz-Hernández C. M., López-Rosas H.,Benítez-Pardo D. y Travieso-Bello A., C. 2007. La topografía y elhidroperíodo: dos factores que condicionan la restauración de los humedalescosteros. Sociedad, Botánica de México, 80, 33-47.

GobiernoMunicipaldeAcapulco(GMA). 2015. Guía Turística Destinos de México Acapulco. from Secretaría de Turismo de Acapulco http://acapulco.gob.mx/wp-content/uploads/2017/06/Acapulco_Guia_Turistica.pdf

Gómez-Baggethun y Groot, R. d. 2007. Capital natural y funciones de los ecosistemas: explorando las bases ecológicas de la economía. Ecosistemas 16(3), 4-14.

Hernández-Trejo, V., Avilés-Polanco, G., y Almendarez-Hernández, M. 2012. Beneficios económicos de los servicios recreativos provistos por la biodiversidad acuática del Parque Nacional Archipiélago Espíritu Santo. Estudios Sociales, 20(40).

Hernández Sampieri, R., Fernández Colado, C., y Baptista Lucio, P. 2014. Metodología de la investigación. (6ta ed.): Mc Graw-Hill.

Heung, V., Qu, H., y Chu, R. 2001. The relationship between vacation factors and socio-demographic and travelling characteristics:the case of Japanese leisure travellers. Tourism Management 22(22), 259-269.

Hsieh, H.-L., Chen, C.-P., y Linc, Y.-Y. 2004. Strategic planning for a wetlands conservation greenway along the west coast of Taiwan. Ocean and Coastal Management, 47(5-6), 257-272.

Huete, R. y Mantecón, A. 2017. La clave es el paisaje. Explorando alternativas al turismo de masas. Arbor, 193(785).

José Luis Rosas-Acevedo, H. Á.-P., Audel Sánchez-Infante, Ana Yolanda Rosas-Acevedo,Sergio García-Ibañez, Laura Sampedro-Rosas, José Guadalupe Granados-Ramírez, Ana Laura Juárez-López. 2014. Índice BMWP, FBI y EPT para determinar la calidad del agua en la laguna de Coyuca de Benítez, Guerrero, México. Revista Iberoamericana de Ciencias, 8.

Labandería, X., León, C., y Vázquez, M. 2007. Economía Ambiental. Madrid: pearson Educación.

Lam, T. y Hsu, C. 2006. Predicting behavioral intention of choosing a travel destination. Tourism Management, 27(4), 589-599.

Lanza, G. d. l., Ruiz, A., Fuentes, P., Camacho, V., Blanco, M., Zamorano, P., . . Arroyo, R. 2013. Propuesta metodológica para la valoración económica en sistemas costeros de México. Investigación Ambiental, 5(1), 7-32.

Lara-Lara, J., Arreola-Lizárraga, J., Calderón-Aguilera, L., Camacho, V., Espino, G. d. l. L., Escofet-Giansone, A., . . Z Zertuche-González, J. 2008. Los ecosistemas costeros, insulares y epicontinentales Capital natural de México, vol. I : Conocimiento actual de la biodiversidad. (pp. 109-134). México: CONABIO.

MA. 2005. Ecosystems and Human Well-Being: Synthesis. Washington, D.C.: Island Press.

Montes, C. y Sala, O. 2007. La Evaluación de los Ecosistemas del Milenio. Las relaciones entre el funcionamiento de los ecosistemas y el bienestar humano. Ecosistemas 16(3), 137-147.

Morales, J. y Pérez, J. L. 2006. Crecimiento poblacional e instrumentos para la regulación ambiental de los asentamientos humanos en los municipios costeros. Gaceta Ecológica, 79, 53-57.

Murphy, L., Benckendorff, P., y Moscardo, G. 2007. Linking Travel Motivation, Tourist Self-Image and Destination Brand Personality. Journal of Travel \& Tourism Marketing, 22(2), 45-59.

Olivera, A. 2011. Patrimonio inmaterial: recurso turístico y espíritu de los territorios. Cuadernos de Turismo, 27, 663-677.

Olmos-Martínez, E., Arizpe-Covarrubias, O., Ibáñez-Pérez, R., y Ortega-Rubio, A. 2015. Servicios ecosistémicos con potencial turístico del parque nacional Archipiélago Espíritu Santo, México. Teoría y Praxis, esp, 158-173.

Ortega-Cabrera, V., Esquivel-Ríos, S., y Ramírez-Hernández, O. 2017. El turismo como factor de impulso para la inclusión del valor de los servicios ecosistémicos en las políticas públicas. El caso de la Reserva de la Biósfera mariposa monarca, México. Turismo y patrimonio, 11. 
Piedragil, G. y Mejía, M. 2001. Análisis sobre la distribución y diversidad del sistema lagunar Coyuca de Benitez y su principal tributario en el estado de Guerrero, México. Paper presented at the XVI Congreso Nacional de Zoología, Zacatecas, Zac.

Pons, R., Morales, L., y Díaz, Y. 2007. La imagen del destino y el comportamiento de compra del turista. Teoría y Praxis, 3, 89-102.

Ruz-Vargas, M. I. 2014. La vulnerabilidad del paraíso. Doctorado en Desarrollo Regional, Universidad Autónoma de Guerrero, Acapulco, Guerrero.

Ruz Vargas, M. 2014. La Vulnerabilidad del Paraíso. Doctorado en Desarrollo Regional, Universidad Autónoma de Guerrero, Acapulco, Guerrero.

Sanjurjo, E., Cadena, K., y Erbstoesser, I. 2005. Valoración Económica de los Vínculos entre Manglar y Pesquerías. Paper presented at the Congreso Iberoamericano de Desarrollo y Medio Ambiente (CIDMA II) Puebla, México

Sanjurjo, E. y Welsh, S. 2005. Una descripción del valor de los bienes y servicios ambientales prestados por los manglares. Gaceta Ecológica, 74, 54-68.

Sarukhán, J., Koleff, P., Carabias, J., Soberón, J., Dirzo, R., Llorente-Bousquets, J., . . Maza, J. d. 1. 2009. Capital Natural de México. Síntesis: conocimiento actual, evaluación y perspectivas de sustentabilidad. México: Comisión Nacional para el Conocimiento y Uso de la Biodiversidad.

SECTUR. 2004. Guía para el diseño y operación de senderos interpretativos. Serie Turismo Alternativo, Fascículo 05, 148.

SIAP. 2018. Atlas Agroalimentario 2012-2018. México: Servicio de Información Alimentaria y Pesquera Secretaría de Agriculura, Ganadería, Desarrollo Rural, Pesca y Alimentación SAGARPA.

Solís-Radilla, M., Hernández-Lobato, L., y Villagómez-Méndez, J. 2016. El valor percibido del destino turístico en relación con el perfil del turista en Acapulco, Guerrero-México. Investigación Administrativa, 46(118).

Strand, I. 2000. Modeling Demand for and Economic Value of Beaches and Their Characteristics: Residential Use in Barbados.

Tallis, H., Money, H., andelman, S., Balvanera, P., Cramer, W., Karp, D., . . Walz, D. 2012. A Global System for Monitoring

Ecosystem Service Change. BioScience, 62(11).

Velázquez, M. 2013. La formulación de las políticas públicas de turismo en México. El caso del programa federal "Pueblos Mágicos" 2001-2012. Diálogos Latinoamericanos, 21, 89-110.

Verissimo, D., MacMillan, D. C., y Smith., R. J. 2011. Toward a systematic approach for identifying conservation flagships Conservation Letters 4, 1-8.

Verona-Ezcurra, A. J. y Rodríguez-Castillo, A. 2013. Valoración económica de bienes y servicios ambientales de la Laguna Conache, Laredo (La Libertad, Perú). REBIOLEST. Revista Científica de Estudiantes, 1(1), 54-70.

Viñals-Vlasco, M. J., Morant-Gonzalez, M., y Quintana, R. 2011. Análisis de los criterios para la valoración turística del patrimonio natural. Investigaciones Turística, 1, 37-50.

Recibido:

$10 / 09 / 2019$

Reenviado:

$18 / 04 / 2020$

Aceptado:

$20 / 05 / 2020$

Sometido a evaluación por pares anónimos 